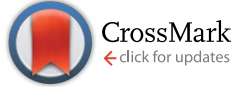

Cite this: J. Anal. At. Spectrom., 2015, 30, 2516

Received 8th September 2015 Accepted 29th October 2015

DOI: $10.1039 / c 5 j a 00382 b$

www.rsc.org/jaas

\section{Multiple ion counting measurement strategies by SIMS - a case study from nuclear safeguards and forensics}

\begin{abstract}
P. M. L. Hedberg, ${ }^{\text {a }}$ P. Peres, ${ }^{b}$ F. Fernandes ${ }^{b}$ and L. Renaud ${ }^{b}$
A primary tool of nuclear safeguards authorities for detecting undeclared nuclear activities is environmental sample analysis containing uranium-bearing aerosol particles. SIMS (Secondary lon Mass Spectrometry) has been established as a powerful technique for performing uranium isotope analysis on safeguards samples. Typically, a screening of the sample for locating the particles of interest is followed by micro-beam measurements of individual particles in order to obtain their precise isotopic composition. The uncertainty of these measurements, in particular for the minor isotopes ${ }^{234} U$ and ${ }^{236} U$, is mainly limited by the counting statistics as the total amount of uranium available is very low, usually less than $1 \mathrm{pg}$. This uncertainty can be reduced when recording several isotopes simultaneously using a multi-collection system. This has been available commercially for many years and has been used for different applications in geosciences on Large Geometry-SIMS (LG-SIMS) instruments. This paper will describe and discuss how isotope measurements of low content uranium materials can be optimized using a multi-ion counting system consisting of five discrete dynode electron multiplier (EM) detectors. The results obtained for uranium isotope ratios are presented for different measurement protocols: static multicollector, dynamic multi-collector and single collector measurements. Some of the fundamental properties of the detector system that affect the overall measurement uncertainty are discussed. Best practices are also suggested based on the current experience.
\end{abstract}

\section{Introduction}

When nuclear uranium materials are processed in industrial quantities, it is difficult to avoid the release to the immediate environment of aerosol particles containing the isotopic signature of the handled materials. This allows safeguards authorities with specialized sampling techniques and analytical laboratories to monitor the handling of nuclear materials at nuclear facilities and to perform forensic investigations to find the origin of materials. ${ }^{\mathbf{1}, 2}$

These analyses are predominantly performed today by SIMS or fission track ${ }^{3-5}$ combined with TIMS (Thermal Ionization Mass Spectrometry) though other techniques employing different combinations of methods and instrumentation are also used. ${ }^{6-10}$

Recently, the performance of SIMS instrumentation for nuclear particle analysis has greatly improved thanks to the implementation of Large Geometry-SIMS (LG-SIMS), ${ }^{\mathbf{1 1}}$ the CAMECA IMS 1280 or 1280-HR, rather than the previously used

${ }^{a}$ European Commission, Joint Research Centre (JRC), Institute for Transuranium Elements, P.O. Box 2340, D-76125 Karlsruhe, Germany. E-mail: magnus.hedberg@ ec.europa.eu

${ }^{b}$ CAMECA, 29 Quai des Grésillons, 92622 Gennevilliers-Cedex, France. E-mail: paula. peres@ametek.com; firmino.fernandes@ametek.com; ludovic.renaud@ametek.com
Small Geometry-SIMS (SG-SIMS), the CAMECA IMS xF series. LG-SIMS instruments feature numerous instrumental advantages, in particular removal of background interferences with minimal loss of transmission and parallel detection of all $\mathrm{U}$ isotopes using the multi-collection system. In addition, the productivity has significantly increased with the implementation of automated particle measurement (APM) software that performs fast screening of the sample to determine the exact location of the uranium particles and their enrichment level. ${ }^{12,13}$

In this paper we describe the performance obtained using the multi-ion counting system equipped with EM detectors for isotopic micro-beam measurements on individual particles. The use of a multi-ion counting system for the APM screening measurements has been described earlier and is not further discussed here. ${ }^{12}$ Some of the basic parameters of the EM detectors like HV adjustment, detector drift and aging effects, as well as detector inter-calibration methods are discussed in the context of improving the isotope measurement quality. Performance obtained using different measurement protocols (static multi-collection, dynamic multi-collection and monocollection) is compared for uranium particle analysis, and advantages and disadvantages of each method are discussed.

Though discussions in this paper are focused on the nuclear safeguards and forensics application, the findings regarding the EM detector behaviour are also valid for other fields of work 
(e.g. in geo/cosmochemistry) and for other types of instruments (e.g. single or multi-collector TIMS and ICPMS - Inductively Coupled Plasma Mass Spectrometry).

\section{Analytical methods and materials}

\section{A. Instrumentation}

The LG-SIMS measurements were performed on a CAMECA IMS 1280-HR at the Institute for Transuranium Elements (ITU) in Karlsruhe, Germany. The IMS $1280-\mathrm{HR}$ is a large geometry double focusing magnetic sector secondary ion mass spectrometer (magnet radius of $585 \mathrm{~mm}$ ). The instrument can operate in both microprobe and microscope modes, since it provides scanning ion images as well as mass filtered stigmatic ion images.

The instrument is equipped with a multi-collection detector system, which allows simultaneous isotope measurements for elements from lithium up to uranium, see Fig. 1. This system consists of five moveable collector units (L2, L1, C, H1, and H2). Each unit is equipped with a set of three slits for adjusting the mass resolution conditions, and with either a discrete dynode electron multiplier (EM) or a Faraday cup (FC) detector for higher signal intensities. For all measurements reported in this paper, the five collector units were equipped with EM detectors (Hamamatsu R4146). A voltage was applied to the DSP2 stigmator after the magnet to increase the mass dispersion in order to measure the ${ }^{234} \mathrm{U},{ }^{235} \mathrm{U},{ }^{236} \mathrm{U},{ }^{238} \mathrm{U}$ and ${ }^{238} \mathrm{U}^{1} \mathrm{H}$ species simultaneously on the five multi-collection EM detectors. The

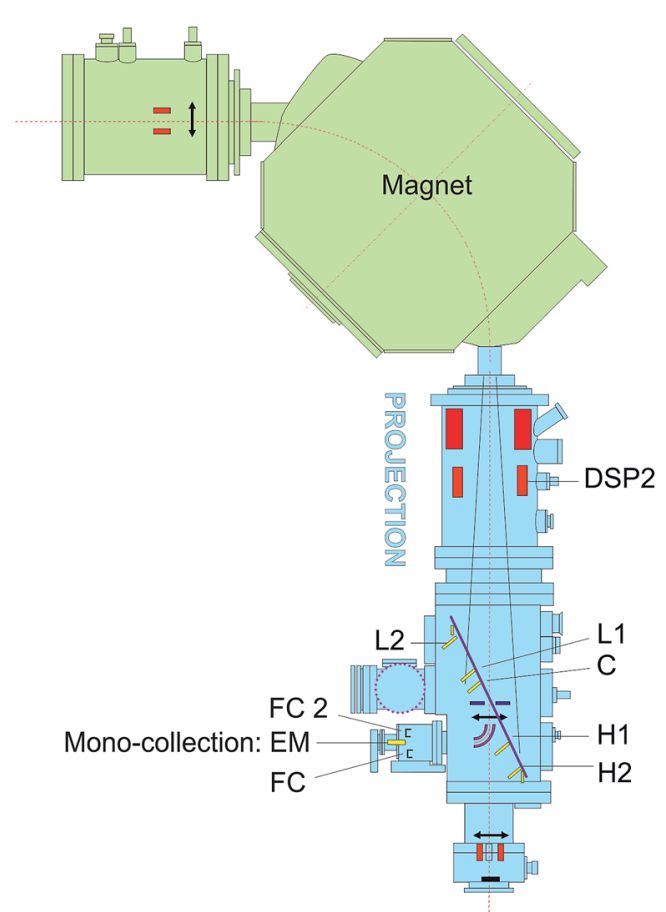

Fig. 1 CAMECA IMS 1280-HR ion optics, a projection area with a detection system after the magnet. DSP2 is an octopole device that is used both as a stigmator and a deflector. It can be used to increase the mass dispersion.

instrument is also equipped with a mono-collection detector system with two FCs and one EM detector (AF150H from ETP Electron Multipliers).

Previous studies on $\mathrm{U}$ particle analyses have shown that the ion yield is higher using negative oxygen primary ion beam $\left(\mathrm{O}^{-}\right.$or $\left.\mathrm{O}_{2}{ }^{-}\right)$compared to positive oxygen primary mode $\left(\mathrm{O}_{2}{ }^{+}\right) \cdot{ }^{11}$ However, positive mode has been selected for the present measurements because of the higher primary beam density and current stability. The selected operating conditions for the measurements are shown in Table 1.

\section{B. Reference materials}

Uranium Certified Reference Materials (CRM) U010 and U100 from New Brunswick Laboratory (DOE, USA) were used for mass calibration and mass bias determinations, as well as for determining the precision and accuracy of uranium isotope measurements. ${ }^{235} \mathrm{U}$ enrichment is $\sim 1$ atom $\%$ for CRM 010 and $\sim 10$ atom $\%$ for CRM U100. ${ }^{234} \mathrm{U}$ and ${ }^{236} \mathrm{U}$ abundances for CRM U100 are higher than that for CRM 010 (more than 10 times for ${ }^{234} \mathrm{U}$, and more than 5 times for $\left.{ }^{236} \mathrm{U}\right) \cdot{ }^{14}$ The standards were prepared in a clean room by transferring the particles onto a planchet (SPI Pyrolitic Graphite Planchet, $25.4 \times 3.2 \mathrm{~mm}$, \#428GP-AB) using a vacuum impactor. ${ }^{15}$

\section{Isotopic micro-beam measurements of uranium particles}

Micro-beam measurements were performed on individual particles using a small focused primary beam rastered over a $10 \times 10 \mu \mathrm{m}$ area around each particle. Uranium isotope ratios are corrected for detector dead time and mass bias. A hydride correction is also applied to the ${ }^{236} \mathrm{U} /{ }^{238} \mathrm{U}$ ratio. ${ }^{11}$

The detector dead time was measured by setting the preamplifier threshold to zero which saturates the pulse counting system as all noise pulses are counted. The inverse of this maximum count rate gives an estimate of the dead time value, see values in Table 2 .

Table 1 Secondary ion mass spectrometry analytical conditions (IMS 1280-HR)

Operating conditions

Primary ions

Primary acceleration voltage

Secondary acceleration voltage

Total incident energy

Optical mode

Primary ion currents for micro-beam

measurements

Image field for transfer optics

Contrast aperture

Field aperture

Entrance slit

Exit slit mono-collection

Exit slit multi-collection

Mass resolution mono-collection (10\%)

Mass resolution multi-collection (10\%)

Energy bandwidth

$\mathrm{O}_{2}^{+}$
$+15 \mathrm{kV}$
$+8 \mathrm{kV}$
$7 \mathrm{keV}$
Circular
$100-300 \mathrm{pA}$

$80 \mu \mathrm{m}$
$400 \mu \mathrm{m}$
$5000 \mu \mathrm{m}$
$174 \mu \mathrm{m}$
$400 \mu \mathrm{m}$
$400 \mu \mathrm{m}$
$\sim 2500$
$\sim 2500$
$30 \mathrm{eV}$

$\mathrm{O}_{2}$

$+8 \mathrm{kV}$

$7 \mathrm{keV}$

Circular

$80 \mu \mathrm{m}$

$400 \mu \mathrm{m}$

$5000 \mu \mathrm{m}$

$174 \mu \mathrm{m}$

$400 \mu \mathrm{m}$

$\sim 2500$

$30 \mathrm{eV}$ 
Table 2 Detector parameters during measurement period, ETP = single detector, L2, L1, C, H1, and H2 = Hamamatsu multi-collection detectors

\begin{tabular}{lllll}
\hline Detector & $\begin{array}{l}\text { Dead time } \\
(\mathrm{ns})\end{array}$ & $\begin{array}{l}\text { High voltage } \\
(\mathrm{V})\end{array}$ & $\begin{array}{l}\text { Yield static } \\
\text { measurements }\end{array}$ & $\begin{array}{l}\text { Yield dynamic } \\
\text { measurements }\end{array}$ \\
\hline ETP & 31 & $\sim 2200$ & - & - \\
L2 & 54.7 & $\sim 2040$ & 0.9994 & 0.9900 \\
L1 & 53.8 & $\sim 1770$ & 1.0000 & 0.9960 \\
C & 54.1 & $\sim 1660$ & 1.0154 & 1.0130 \\
H1 & 55.3 & $\sim 2133$ & 1.0000 & 1.0000 \\
H2 & 55.9 & $\sim 1600$ & 1.0145 & 1.0071 \\
\hline
\end{tabular}

The detector dark noise was measured at mass 5 for a period of 60 minutes. The dark noise was in the range of $2.8 \times 10^{-4}$ count per $s$ to $3.3 \times 10^{-3}$ count per $s$ for the 5 Hamamatsu detectors. The single collection EM had a dark noise of $2 \times 10^{-3}$ count per s. For the measurements reported in this paper there was no subtraction of detector dark noise.

Multi-collector measurements are also corrected for the detector yield values reported in Table 2, whereas a drift time correction is applied to single collector measurements. ${ }^{\mathbf{1 6}}$

In this paper, uranium isotope data are presented in atom\%, as commonly used in safeguards applications.

Three different measurement protocols have been investigated in this paper. The selected settings for these measurements are described in Table 3:

(a) Peak jump (PJ) mono-collection measurements where ${ }^{234} \mathrm{U}$, ${ }^{235} \mathrm{U},{ }^{236} \mathrm{U},{ }^{238} \mathrm{U}$ and ${ }^{238} \mathrm{U}^{1} \mathrm{H}$ species are recorded sequentially on the EM ETP detector. The mass peak switching is performed by changing the magnetic field.

(b) Static multi-collection measurements using the five EM detectors (L2, L1, C, H1, and $\mathrm{H} 2$ ) for simultaneous measurements of ${ }^{234} \mathrm{U},{ }^{235} \mathrm{U},{ }^{236} \mathrm{U},{ }^{238} \mathrm{U}$ and ${ }^{238} \mathrm{U}^{1} \mathrm{H}$, see Fig. 2.

(c) Dynamic multi-collection measurements consisting of simultaneous isotope detection as in (b), combined with magnetic peak jumping for measuring ${ }^{235} \mathrm{U}$ and ${ }^{238} \mathrm{U}$ species using the same detector (L1), see Fig. 2.

\section{Fundamentals of the EM detection system}

EM detectors work in a direct pulse counting mode. A secondary ion striking the first dynode (conversion dynode) of the EM induces secondary electron emission. These electrons are then

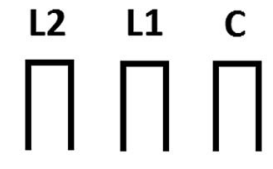

Sequence $12340 \quad 235 U \quad 236 U$

Sequence 2

$238 U$

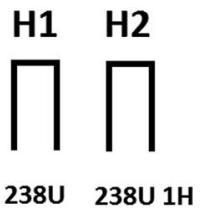

Fig. 2 For static multi-collection measurements only sequence 1 is performed. For dynamic multi-collection measurements, the ${ }^{234} \mathrm{U} /{ }^{238} \mathrm{U},{ }^{236} \mathrm{U} / /^{238} \mathrm{U}$ and ${ }^{238} \mathrm{U}^{1} \mathrm{H} / /^{238} \mathrm{U}$ isotope ratios are computed from signals measured during sequence 1 , whereas the ${ }^{235} \mathrm{U} /{ }^{238} \mathrm{U}$ ratio is obtained from signals recorded on $\mathrm{L} 1$ detector, sequences $1\left({ }^{235} \mathrm{U}\right)$ and $2\left({ }^{238} \mathrm{U}\right)$.

accelerated and multiplied through the successive dynode stages of the EM. The gain of the detector is defined as the mean number of output electrons per secondary ion (typically in the range of $10^{8}$ ). For most of the secondary ions reaching the detector, a charge pulse is produced at the last dynode output. A preamplifier converts the charge pulses into voltage pulses and amplifies them. Then a discriminator compares the amplified voltage pulses to a given detection threshold and rejects the smaller pulses to cut off the system noise. The amplitude of the output voltage pulses is not constant but distributed according to a pulse-height distribution (PHD), see Fig. 3.

It is known that for EM detectors, the gain of the detector can be affected by short term drift issues and long term aging phenomena. Below is a list of three main components of the detector drift and aging:

(1) Long term aging of the last dynodes that needs to be compensated for by progressively increasing the EM high voltage throughout the EM's lifetime in order to keep the gain constant. The lifetime of an electron multiplier depends on the total number of ions collected (total integrated charge), therefore, frequent high intensity measurements shorten the EM lifetime. The vacuum environment also plays a major role in determining the overall life of the detector. This deterioration is known to be caused by carbon deposition on the last dynodes. ${ }^{\mathbf{1 7}}$

(2) Long term deterioration of the first dynode due to deposition of impinging ions, see Fig. 4 and 5. As the detector gets older, this effect can be observed by scanning the ion beam along $X$ and $Y$ directions over the detector first dynode, as the gain becomes lower at the usual position where the ion beam hits the detector. This effect is reduced in time if the impinging

Table 3 Time settings for the different measurement protocols. The total acquisition time includes the waiting times required for magnetic field stabilisation

\begin{tabular}{ll}
\hline Method & Settings \\
\hline PJ mono-collection measurements & Integration time sequence: ${ }^{234} \mathrm{U}=4 \mathrm{~s},{ }^{235} \mathrm{U}=2 \mathrm{~s},{ }^{236} \mathrm{U}=4 \mathrm{~s},{ }^{238} \mathrm{U}=2 \mathrm{~s}$, and ${ }^{238} \mathrm{U}^{1} \mathrm{H}=2 \mathrm{~s}$ \\
Static multi-collection measurements & Total acquisition time $=768 \mathrm{~s}(40 \mathrm{cycles})$ \\
& Integration time $=4 \mathrm{~s}$ \\
Dynamic multi-collection measurements & Total acquisition time $=705 \mathrm{~s}(167$ cycles $)$ \\
& Integration time for sequence $1=4 \mathrm{~s}$ and for sequence $2=1 \mathrm{~s}$ \\
& Total acquisition time $=710 \mathrm{~s}(100$ cycles $)$
\end{tabular}




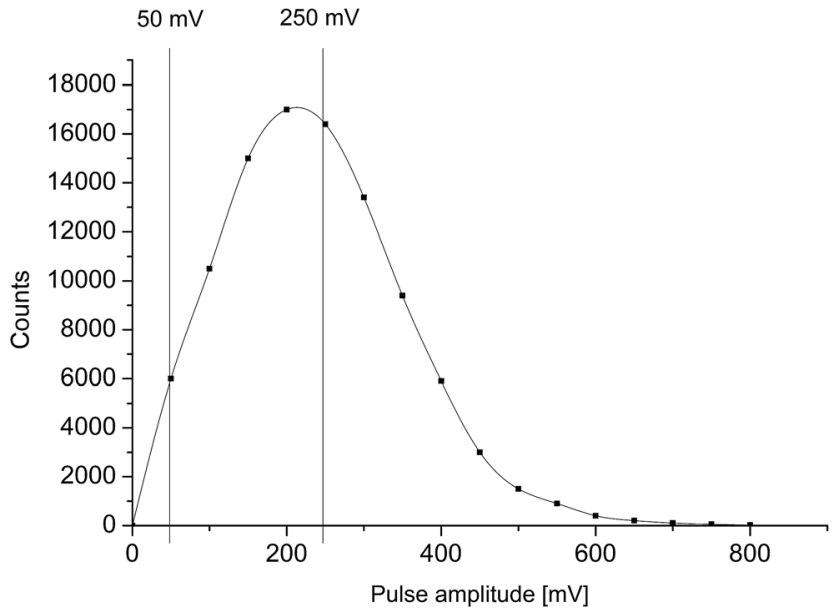

Fig. 3 Typical detector pulse height distribution (PHD) from a Hamamatsu EM detector. Thr1 and Thr2 pulse amplitude threshold values used by the EM HV routine are typically set at $50 \mathrm{mV}$ and $250 \mathrm{mV}$, respectively.

ion beam is enlarged or scanned over the first dynode. ${ }^{18}$ In our case, after a deterioration was observed, the lifetime of the detectors has been increased by shifting the ion beam along $Y$ so that it hits a fresh area on the first dynode, see Fig. 6 . This can be done by applying a small offset along the $Y$ direction using main deflector DSP2. This brings back a correct mass peak flatness along $X$ and also slightly improves the detector yield, resulting in a lower EM HV.

(3) Short term drift behaviour where the detector yield changes depending on the intensity of the applied ion beam and drifts back to its original position if left without an ion beam. Interestingly, this drift is in the opposite direction compared to the long term aging effect. As this effect is rarely discussed or described, particular attention is given to it in this paper. A previous study showed that detector

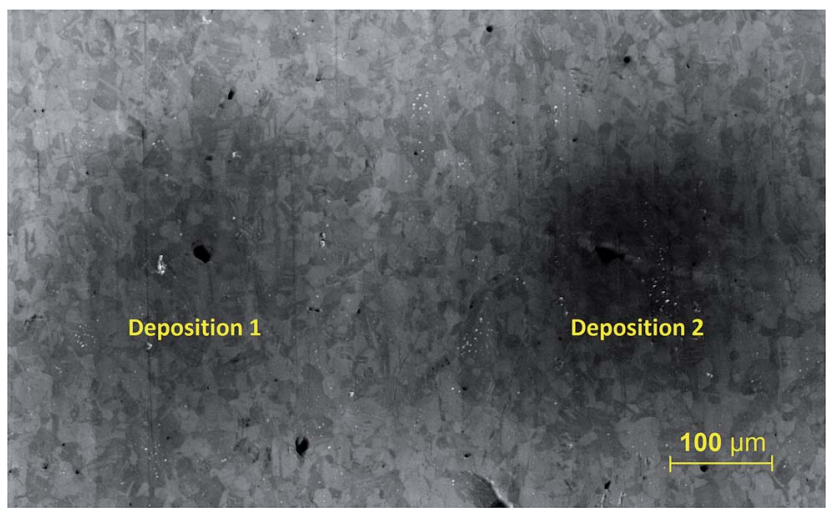

Fig. 4 Scanning electron microscope image on the first dynode of a Hamamatsu detector. Two dark areas of approximately 300-400 $\mu \mathrm{m}$ in diameter are observed, most likely a slight charging effect due to a deposition of material that is less conductive. Two different ion beam $Y$ impact positions have been used on this detector, which explains the presence of two deposition areas. The horizontal axis of this image corresponds to the $Y$ direction of the detector.

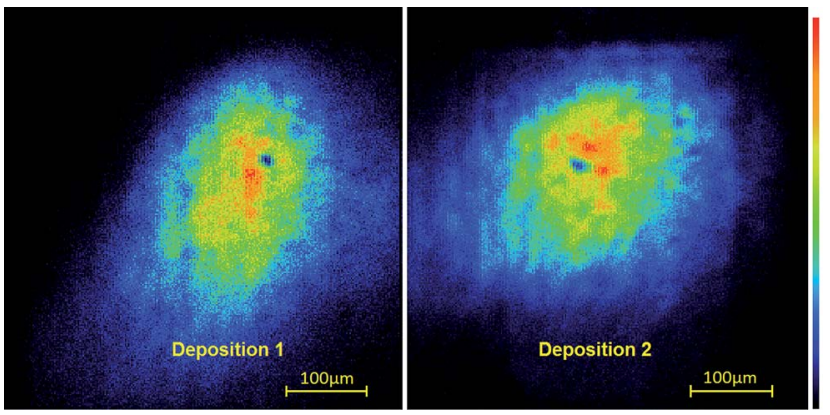

Fig. 5 LG-SIMS ${ }^{238} \mathrm{U}$ scanning ion images on deposition area 1 and deposition area 2 shown in Fig. 4, showing that the depositions contain uranium.

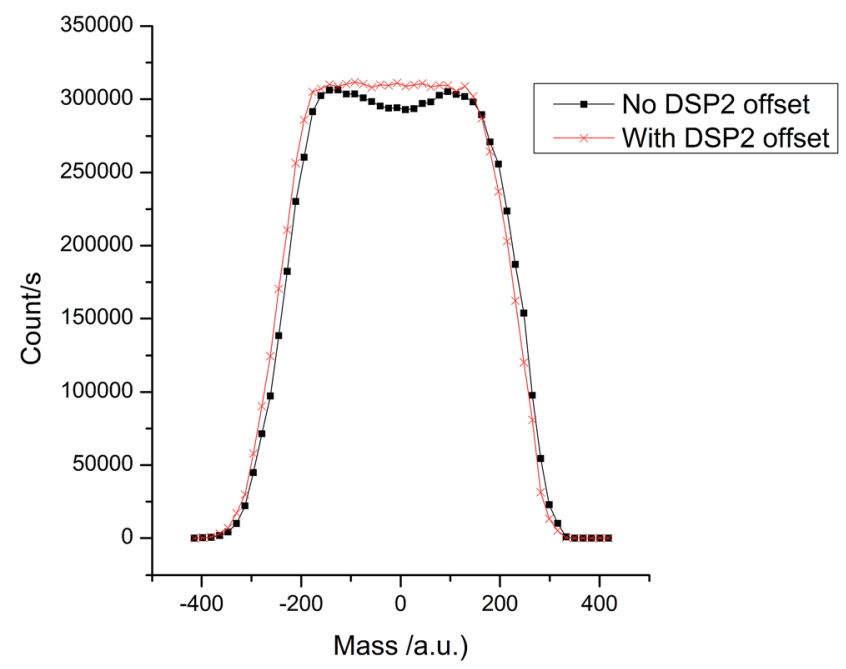

Fig. 6 Mass scan at mass ${ }^{238} U$ with and without an offset on the DSP2 deflector, which corresponds to two different $Y$ positions for the ion beam impact on the first dynode. A significantly better peak flatness is obtained using a new impact position (with DSP2 offset).

non-linearity is affected when switching a high intensity beam into a detector used for lower intensity measurements. ${ }^{19}$ This could possibly be related to short term drift issues. Examples are shown below of different short term drift measurements. Experience shows that the amplitude and duration of the short term drift depends on the detector age. When the detector is new, it shows a fairly high degree of short term yield drift, and its stability improves as it gets older. When a highly stable detector is required one can age a new detector by sending a high beam dose over the first dynode until it stabilises.

In CAMECA instruments, a quick iterative automated routine is used to adjust the EM HV in order to keep the PHD curve within a given template. Practically, the routine does not process the PHD curve, but adjusts EM HV so that when the EM threshold is set to Thr2, typically $250 \mathrm{mV}$, the signal is half of the signal obtained using Thr1, typically $50 \mathrm{mV}$, see Fig. 3. Generally, Thr1 corresponds to the working threshold for removing noise pulses and is kept fixed all along the EM lifetime. 
This automatic HV adjustment routine was recently included in the analysis acquisition software, and thus can be performed at the start of each micro-beam measurement. The first results obtained using this new software capability are shown in Section B.

For uranium measurements using a high sample extraction voltage ( $8 \mathrm{kV}$ for the present measurements), new multi-collection Hamamatsu detectors typically start with a voltage at about $1600 \mathrm{~V}$ which gradually increases up to about $2200-2400 \mathrm{~V}$. The starting voltage for the mono-collection ETP detector is typically higher around $1850 \mathrm{~V}$. The EM HV values used for the present measurements are shown in Table 2.

\section{Results and discussion}

\section{A. Short term drift measurements}

Fig. 7 shows a short term drift pattern recorded for the single ETP EM using the automatic EM high voltage (EM HV) routine. The voltage values are the result of repeated EM HV adjustments. The drift pattern was measured after a period of about 14 hours with no signal on the detector. At first, there is a rapid detector drift corresponding to a voltage drop, and then the detector stabilises. The detector drifts back within about an hour to the starting HV value after the ion beam is switched off.

A similar test is shown in Fig. 8 for a new Hamamatsu detector, using two different ion beam intensities. The results show that the short term drift increases with the ion beam intensity.

The detector drift can be measured more precisely by physically connecting the detector to two pulse counting channels in parallel. One channel is set to the normal $50 \mathrm{mV}$ threshold (Thr1) and the second channel set at $250 \mathrm{mV}$ (Thr2). The ratio of the intensities $I_{\mathrm{Thr} 1} / I_{\mathrm{Thr} 2}$ provides a continuous measurement of the PHD shift, similarly to what is performed by the CAMECA EM HV adjustment routine. For short term drift, the threshold ratio usually drops below the expected value around 2, which

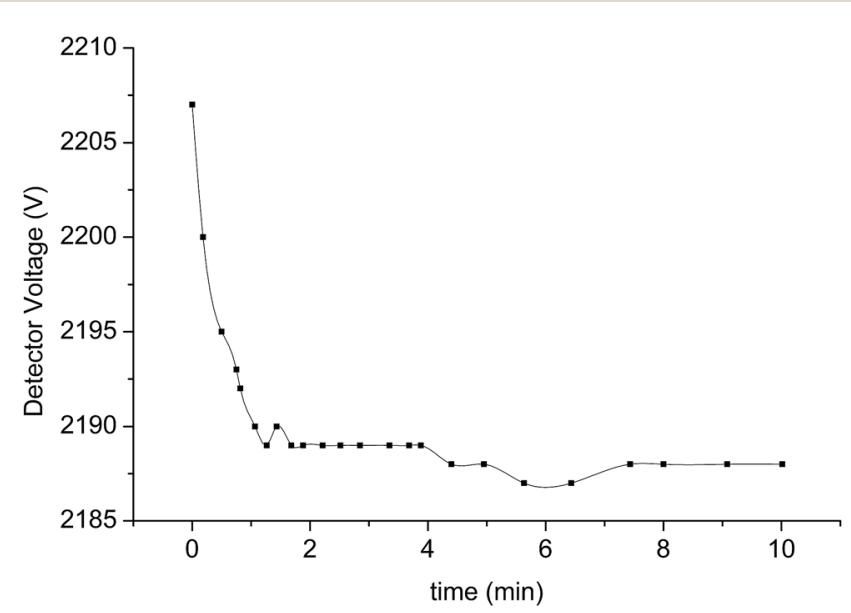

Fig. 7 Drift pattern obtained by performing repeated EM HV adjustments for the ETP detector of the mono-collection system, using $a^{238} \mathrm{U}$ ion beam intensity of $325 \mathrm{kcts} \mathrm{s}^{-1}$. The detector stabilises after $80 \mathrm{~s}$ and a voltage decrease of $19 \mathrm{~V}$. This detector has gone through a significant aging thus the voltage is rather high.

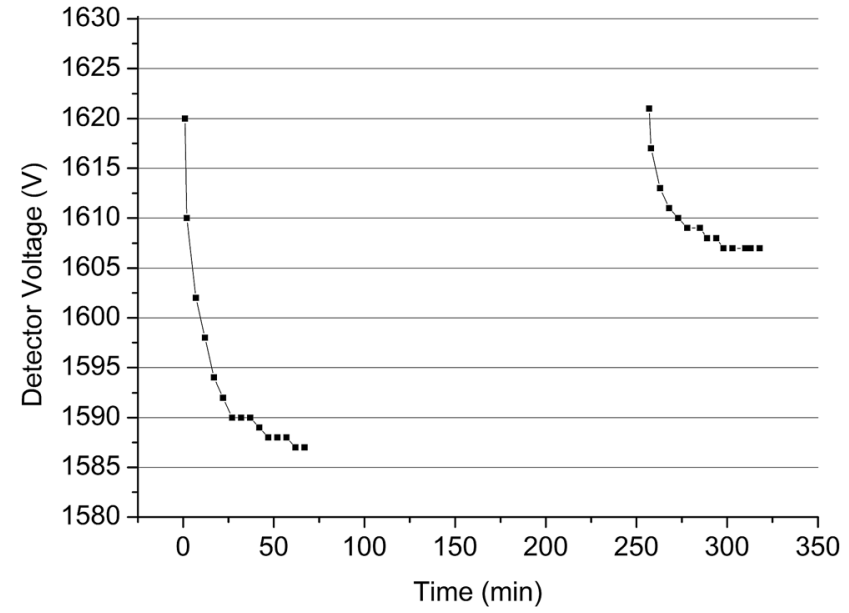

Fig. 8 Drift patterns for an almost new Hamamatsu detector at two different ion beam intensities. The first drift curve is obtained using $670 \mathrm{kcts} \mathrm{s}^{-1}$ and the second with $170 \mathrm{kcts} \mathrm{s}^{-1}$. The time between the two measurements (with no impinging ion beam) allowed the detector to drift back to its previous voltage.

means the pulse height distribution moves towards high amplitude values. This is equivalent to an EM HV increase so that the short term drift must be compensated by decreasing the EM voltage, as shown in Fig. 7 and 8.

In Fig. 9, measurements using parallel counting channels with different thresholds have been performed on two detectors, C (almost new) and H1 (significantly aged). The drift measurements show that the new detector (C) drifts significantly more and for a longer period of time compared to the older one (H1).

Fig. 10 shows similar drift measurements performed on detectors $\mathrm{L} 1 \quad\left({ }^{235} \mathrm{U}\right)$ and $\mathrm{H} 1 \quad\left({ }^{238} \mathrm{U}\right)$ while simultaneously measuring the isotopic ratio ${ }^{235} \mathrm{U} /{ }^{238} \mathrm{U}$ on $\mathrm{CRM} \mathrm{U} 100$, as in Fig. 11. Fig. 10 shows that detector L1 drifts more than H1 despite a signal 10 times lower $\left({ }^{238} \mathrm{U}\right.$ count rate of $\left.\sim 300 \mathrm{kcts} \mathrm{s}^{-1}\right)$.

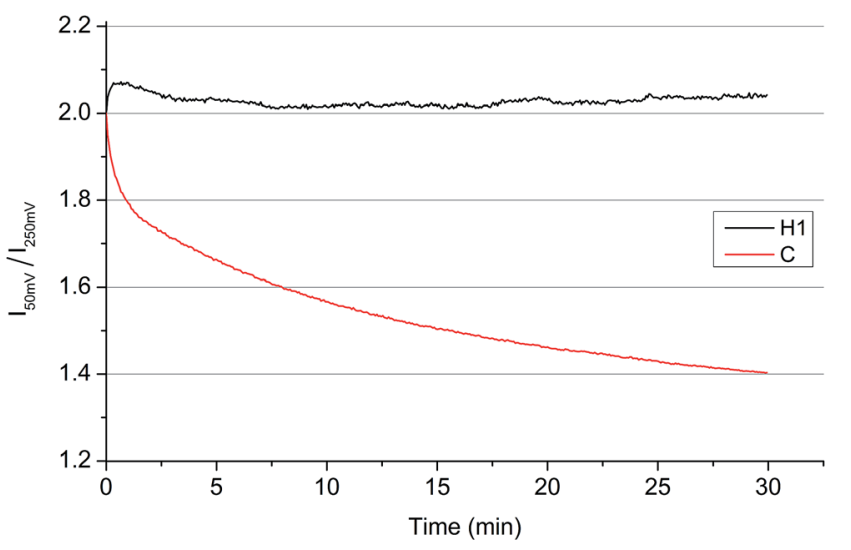

Fig. 9 Drift patterns for detectors $\mathrm{C}$ and $\mathrm{H} 1$ obtained from the intensity ratio for two different threshold values $I_{50 \mathrm{mv}} / I_{250 \mathrm{mv}}$, using $a^{238} \mathrm{U}$ count rate around $300 \mathrm{kcts} \mathrm{s}^{-1}$ for both detectors. The intensity ratio of detector $C$ can be brought back to its original value by reducing the EM voltage of about $\sim 35 \mathrm{~V}$. 


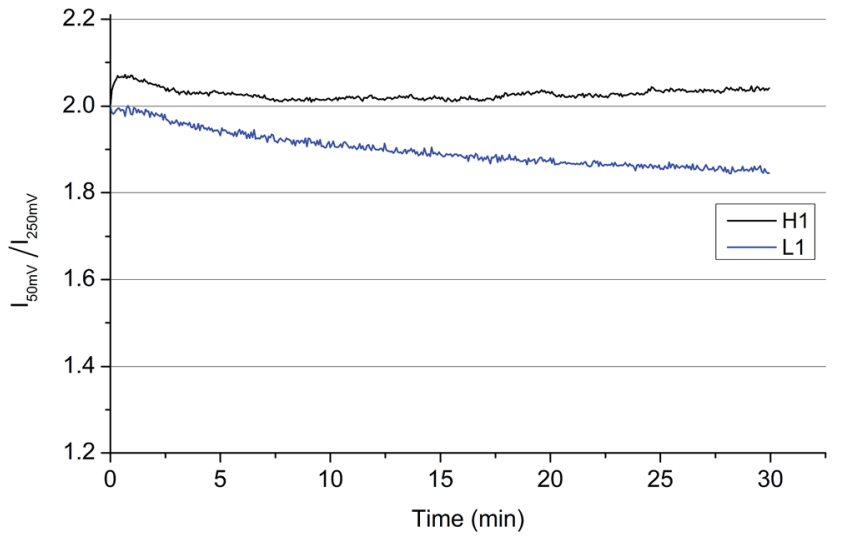

Fig. 10 Drift patterns for detectors $\mathrm{L} 1\left({ }^{235} \mathrm{U}\right)$ and $\mathrm{H} 1\left({ }^{238} \mathrm{U}\right)$ were obtained while measuring simultaneously the ${ }^{235} \mathrm{U} / 2^{238} \mathrm{U}$ ratio on CRM U100, see Fig. 11. The count rate was $\sim 300 \mathrm{kcts} \mathrm{s}^{-1}$ on the $\mathrm{H} 1\left({ }^{238} \mathrm{U}\right)$ detector.

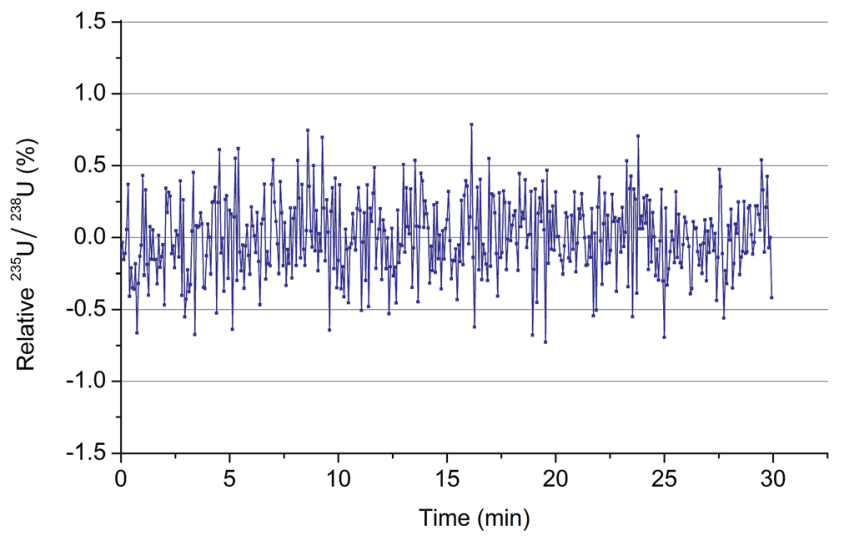

Fig. 11 Relative ${ }^{235} U /{ }^{238} \mathrm{U}$ deviation from the average value in multicollection ratio measurement performed while recording the detector drift patterns shown in Fig. 10. Each data point corresponds to a measurement of 4 seconds.

A linear fit to the ${ }^{235} \mathrm{U} /{ }^{238} \mathrm{U}$ data in Fig. 11 indicates however that the isotope ratio drift is small, not larger than $\sim 0.5 \%$ during the 30 minutes of measurement. A long series of measurements would require repeated $\mathrm{EM} \mathrm{HV}$ adjustments in-between the measurements to compensate for the detector drift. This has been confirmed by a 10 hour long measurement session on the CRM U100 sample, using the auto EM HV adjust function for detector $\mathrm{H} 1\left({ }^{238} \mathrm{U}\right.$, count rate of $\left.\sim 130 \mathrm{kcts} \mathrm{s}^{-1}\right)$ but not for L1 $\left({ }^{235} \mathrm{U}\right)$. This set of measurements showed an overall increase in the ${ }^{235} \mathrm{U} /{ }^{238} \mathrm{U}$ ratio of $3 \%$ over the total 10 hours.

It is important to take these short term drift patterns into account, in particular when performing multi-collection analyses. For uranium measurements one should avoid putting high intensity beams on the minor isotope detectors prior to sample measurements as this will push the detector away from its normal working yield. The $\mathrm{C}$ detector which presents a large drift (Fig. 9) can be used for the measurement of the ${ }^{236} \mathrm{U}$ minor isotope, as the drift is expected to be negligible for such a low intensity signal. The detector used for the ${ }^{238} \mathrm{U}$ measurements has a very good stability making it well suitable for the measurements at high intensities. The drift patterns have also to be taken into account for selecting the method for detector yield inter-calibration. As an example, sequentially measuring a given high intensity ion beam on the different detectors does not provide accurate results due to the large difference in ion beam intensities when performing real $U$ isotope measurements. A recommended method for detector yield inter-calibration is described in Section B.2.

\section{B. Isotope measurements on CRM samples}

Sets of uranium isotope ratio measurements on standard particles CRM U010 and CRM U100 are described below. These measurements are well suited for comparing the performance of different analytical protocols as the particles are large and free of any matrix materials.

For all results, "Mean" is the average isotope abundance obtained from many analyses, each analysis being performed on a different particle. "SD" is the absolute standard deviation, $\mathrm{RSD} \%$ the relative standard deviation (in percent), and "Bias\%" the deviation (in percent) from the certified values.

B.1. PJ mono-collection measurements. For the single collector measurements, ${ }^{234} \mathrm{U},{ }^{235} \mathrm{U},{ }^{236} \mathrm{U},{ }^{238} \mathrm{U}$ and ${ }^{238} \mathrm{U}^{1} \mathrm{H}$ species were recorded in a peak jump sequence, see Table 3. Measurements were performed at count rates of 100-350 kcts $\mathrm{s}^{-1}$ on ${ }^{238} \mathrm{U}$. The results are shown in Table 4 for CRM U010 and CRM U100 samples. Data are corrected for a mass bias of -0.00296 per amu. The automatic EM HV adjust routine was not used here because all species are measured on the same detector thus the ratios are not affected by detector yield variations.

As expected, the measurement precision on the CRM U100 is better than on the CRM U010 due to the higher counting rates for ${ }^{234} \mathrm{U},{ }^{235} \mathrm{U}$, and ${ }^{236} \mathrm{U}$ and thus improved Poisson statistics. The measurement uncertainty is mainly determined by the combination of the Poisson statistics and the drift time correction for the ${ }^{235} \mathrm{U}$ measurement. A possible improvement could be foreseen by applying fast electrostatic mass peak switching, in order to reduce the uncertainty component from the drift time correction.

The mono-collection PJ protocol provides good quality data as long as the particles are large enough and the primary beam remains reasonably stable. It should also be pointed out that it is the simplest method to implement.

B.2. Static multi-collection measurements. In these measurements, all isotopes were recorded simultaneously using the multi-collection system, see Table 3 and Fig. 2. Measurements were performed at count rates of $100-350 \mathrm{kcts} \mathrm{s}^{-1}$ on ${ }^{238} \mathrm{U}$. Data are corrected for a mass bias of -0.00232 per amu.

The detector yield for $\mathrm{L} 2\left({ }^{234} \mathrm{U}\right), \mathrm{L} 1\left({ }^{235} \mathrm{U}\right)$ and $\mathrm{C}\left({ }^{236} \mathrm{U}\right)$ versus $\mathrm{H} 1$ $\left({ }^{238} \mathrm{U}\right)$ was derived from a set of CRM U100 measurements by matching the measured values to the nominal isotope ratios. The $\mathrm{H} 2$ detector yield $\left({ }^{238} \mathrm{U}^{1} \mathrm{H}\right)$ versus $\mathrm{H} 1$ was derived from a peak jump measurement using a count rate of about $50 \mathrm{kcts}$. It is important to have the ion beam hitting the first dynode at the same geometrical position for all detectors during these measurements, as any shift will change the relative detector yield. 
Table 4 Results in atom\% from 41 measurements on CRM U010 and 54 measurements on CRM U100 using a single ETP detector. The measurements were made over a period of 2 weeks (U010) and 1 month (U100)

\begin{tabular}{|c|c|c|c|c|c|c|c|c|}
\hline & 234 & 235 & 236 & 238 & 234 & 235 & 236 & 238 \\
\hline SD & 0.0001 & 0.0018 & 0.0002 & 0.0019 & 0.0004 & 0.0144 & 0.0004 & 0.0144 \\
\hline RSD\% & 2.55 & 0.18 & 2.60 & 0.0019 & 0.61 & 0.14 & 1.03 & 0.0160 \\
\hline Bias\% & -0.26 & -0.04 & -0.23 & 0.0004 & -0.03 & 0.03 & 0.35 & -0.0031 \\
\hline
\end{tabular}

The results of the measurements are shown in Table 5 for CRM U010 and CRM U100 samples. For CRM U010 measurements, the automatic EM HV adjustment routine was applied on detector $\mathrm{H} 1\left({ }^{238} \mathrm{U}\right)$. This routine was also applied for CRM U100 measurements, but in this case the auto HV adjust was performed every fifth analysis on detector $\mathrm{L} 1\left({ }^{235} \mathrm{U}\right)$ because of the higher ${ }^{235} \mathrm{U}$ count rate for this sample ( $\sim 10$ times higher). In fact, a series of preliminary measurements performed during a time period of 10 hours on this sample showed that without EM HV adjustment a drift of the L1 detector is observed (see also Section A and Fig. 10 and 11).

The results show that the use of static multi-collector measurements improves the measurement precision compared to single collector measurements, which is mainly due to the improved Poisson statistics (for a similar analysis duration). The auto HV adjust function works well but should be improved in order to allow adjustment of more than one detector for each analysis, typically detectors $\mathrm{L} 1\left({ }^{235} \mathrm{U}\right)$ and $\mathrm{H} 1\left({ }^{238} \mathrm{U}\right)$ that receive most intense signals.

It should be noted that these measurements were performed over short periods of time, thus uncertainties are not affected by long term variations.

B.3. Dynamic multi-collection measurements. For these measurements, all isotopes were measured simultaneously in a first sequence followed by a peak jump in order to measure the ${ }^{235} \mathrm{U}$ and ${ }^{238} \mathrm{U}$ signals using the same detector (L1), see Table 3 and Fig. 2. Measurements were performed at count rates of $100-350 \mathrm{kcts} \mathrm{s}^{-1}$ on ${ }^{238} \mathrm{U}$. Data are corrected for a mass bias of -0.00232 per amu. The automatic EM HV adjust routine was applied for all measurements on detector H1 $\left({ }^{238} \mathrm{U}\right)$. The results are shown in Table 6 for CRM U010 and CRM U100 samples.
These data demonstrate that excellent measurement precision can be maintained over long periods of time for ${ }^{234} \mathrm{U},{ }^{235} \mathrm{U}$ and ${ }^{236} \mathrm{U}$. The precision level for ${ }^{234} \mathrm{U}$ and ${ }^{236} \mathrm{U}$ measurements is slightly worse compared to the static multi-collection protocol but better than that for single collector measurements (for the same analysis duration). This method has the advantage that the ${ }^{235} \mathrm{U} /{ }^{238} \mathrm{U}$ ratio is not affected by detector yield variations since the two isotopes are measured on the same detector.

The dynamic measurement protocol also provides a continuous monitoring of the mass bias stability, whereas separate mass bias measurements are required for static multi-collection mode.

\section{Isotope measurements on real samples}

Interlaboratory comparisons have shown that the results obtained for uranium isotope measurements on standard $\mathrm{U}$ particle materials vary not only between different measurement techniques but also between laboratories using the same technique. ${ }^{20}$ One reason for the spread in quality between laboratories using the same technique could be related to the handling of some of the detector issues presented in this paper.

Measurements on inspection samples at ITU using the SIMS dynamic multi-collection method described above show that measurement uncertainties (1SD) on samples with large particles (micrometers in diameter) are in the range of $\sim 0.1-0.3 \%$ for ${ }^{235} \mathrm{U}$ atom $\%$ and $\sim 0.4-3 \%$ for ${ }^{234} \mathrm{U}$ atom $\%$, in an enrichment range from depleted to $20 \%$. The detection limit for the ${ }^{236} \mathrm{U}$ atom\% depends on the enrichment level due to the hydride correction; detection levels of 1-20 ppm (3SD) are typically obtained in an enrichment range from depleted to $20 \%$. It must be pointed out that the uncertainties obtained on real $U$ particle

Table 5 Results in atom\% from 48 measurements on CRM U010 and 44 measurements on CRM U100. The measurements were made over a period of 2 days for CRM U010. The CRM U100 dataset was recorded during two automated chain measurements performed over a period of five days

\begin{tabular}{|c|c|c|c|c|c|c|c|c|}
\hline & \multicolumn{4}{|c|}{ CRM U010 } & \multicolumn{4}{|c|}{ CRM U100 } \\
\hline & 234 & 235 & 236 & 238 & 234 & 235 & 236 & 238 \\
\hline Mean & 0.0054 & 1.0025 & 0.0069 & 98.9853 & 0.0677 & 10.2011 & 0.0380 & 89.6932 \\
\hline SD & 0.0001 & 0.0017 & 0.0001 & 0.0017 & 0.0003 & 0.0081 & 0.0002 & 0.0082 \\
\hline RSD\% & 1.46 & 0.17 & 1.14 & 0.0017 & 0.45 & 0.08 & 0.49 & 0.0091 \\
\hline Bias\% & 0.05 & -0.11 & 0.35 & 0.0011 & -0.01 & 0.09 & 0.04 & -0.0105 \\
\hline
\end{tabular}


Table 6 Results in atom\% from 68 measurements on CRM U010 and 43 measurements on CRM U100. The measurements were made over a period of 2 months

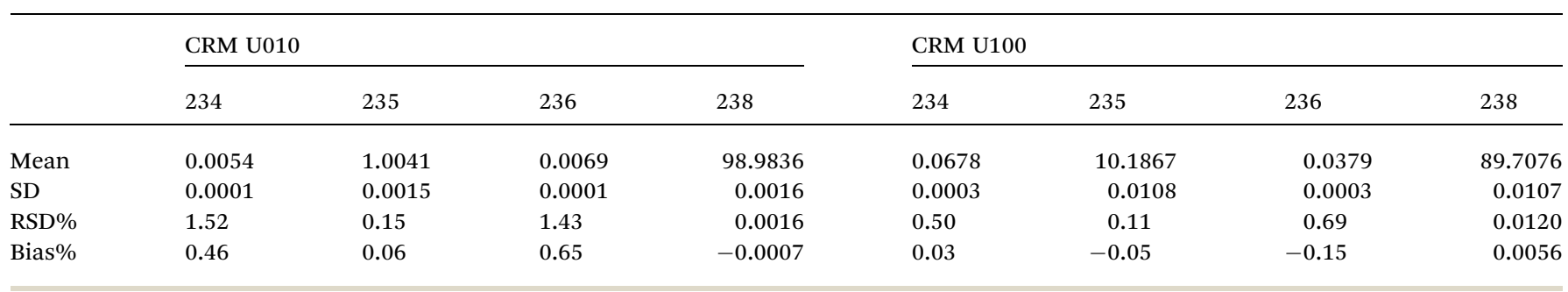

samples vary significantly from sample to sample as the particle sizes are not identical.

\section{Conclusions}

Uranium isotope measurements are challenging because of the large difference in isotopic abundance, and also due to the demand of high precision and accuracy on ${ }^{235} \mathrm{U}$ as well as on the minor isotope measurements.

Parallel detection using multi-collection is preferred to sequential mono-collection mode as the uncertainty determined by Poisson (counting) statistics is significantly reduced for the same analysis time. It should be noted that for particle analysis, the analysis duration is limited by the available particle volume. Therefore, the multi-collection mode provides much better precision for small particles as it maximizes the number of ions collected before the particle is sputtered away.

However, precision using multi-collection may be limited by detector drift and aging effects that require inter-calibration of the detector yields. A recent improvement in controlling the detector drift is the implementation of the automated EM high voltage adjustment during analysis acquisition.

A compromise between the single collector measurements and the static multi-collector measurements is the use of dynamic multi-collector measurements that provides high precision measurements of the ${ }^{235} \mathrm{U} /{ }^{238} \mathrm{U}$ ratio at levels of $1 \%$ in a robust way. The results shown in this paper demonstrate that these measurements provide highly precise and accurate measurements for all $\mathrm{U}$ isotopes.

The static multi-collector measurement protocol has the potential to provide the lowest uncertainties (close to the Poisson statistics) even at high signal intensities if detector drift issues and yield variations can be well controlled and/or corrected. The long term aging and short term drift issues can be handled reasonably well if detectors are sufficiently stable (having gone through some aging); the EM HV adjust is used frequently and detectors are not stressed with high intensities beams prior to measurements at lower intensities. Another problem however is the deterioration of the first dynode with time which in our case seems to be related to the accumulation of uranium on the dynode surface. This is an issue as the detector yield then becomes very sensitive to the precise ion beam shape and position on the first dynode. Defocusing or scanning the beam before it hits the first dynode might be helpful for minimizing the first dynode deterioration; however one would need to carefully verify that this does not affect measurement precision or accuracy.

Studies on EM detector drift effects and suggested measurement protocols based on current experience are presented in this paper. Further investigations are needed to better understand the causes of the short term drift and first dynode deterioration in order to more precisely control or compensate for these effects.

\section{Acknowledgements}

The authors acknowledge the technical assistance of Noelle Albert and Christine Vincent at ITU and their help in preparing the standard planchets and for comments made. The authors also acknowledge Herbert Sigmund's (IAEA Seibersdorf, Austria) for his assistance in some of the detector drift measurements and for comments made.

\section{References}

1 E. Kuhn, D. Fischer and M. Ryjinksi, Environmental sampling for IAEA Safeguards: a five year review, IAEA-SM-367/10/01.

$2 \mathrm{~V}$. Fedchenko, The new nuclear forensics - analysis of nuclear materials for security purposes, SIPRI, Oxford university press, 2015, ISBN 978-0-19-873664-6.

3 C. G. Lee, K. Iguchi, J. Inagawa, D. Suzuki, F. Esaka, M. Magara, S. Sakurai, K. Watanabe and S. Usuda, Development in fission track-thermal ionization mass spectrometry for particle analysis of safeguards environmental samples, J. Radioanal. Nucl. Chem., 2007, 272(2), 299-302, DOI: 10.1007/s10967-007-0519-0.

4 C. G. Lee, D. Suzuki, F. Esaka, M. Magara, N. Shinohara and S. Usuda, Selective Detection of Particles Containing Highly Enriched Uranium for Nuclear Safeguards Environmental Samples, J. Nucl. Sci. Technol., 2009, 46(8), 809-813.

5 F. Esaka, C. G. Lee, M. Magara and T. Kimura, Fission tracksecondary ion mass spectrometry as a tool for detecting the isotopic signature of individual uranium containing particles, Anal. Chim. Acta, 2012, 721, 122-128.

6 F. Esaka, M. Magara, C. G. Lee, S. Sakurai, S. Usuda and N. Shinohara, Comparison of ICP-MS and SIMS techniques for determining uranium isotope ratios in individual particles, Talanta, 2009, 78, 290-294.

7 Z. Varga, Application of laser ablation inductively coupled plasma mass spectrometry for the isotopic analysis of single uranium particles, Anal. Chim. Acta, 2008, 625, 1-7. 
8 F. Pointurier, A. Hubert and A. C. Pottin, Performance of laser ablation: quadrupole-based ICP-MS coupling for the analysis of single micrometric uranium particles, $J$. Radioanal. Nucl. Chem., 2013, 296, 609-616, DOI: 10.1007/ s10967-012-2119-x.

9 S. Kappel, S. F. Boulyga and T. Prohaska, Direct uranium isotope ratio analysis of single micrometer-sized glass particles, J. Environ. Radioact., 2012, 113, 8-15.

10 S. Boulyga, S. Konegger-Kappel, S. Richter and L. Sangély, Mass spectrometric analysis for nuclear safeguards, J. Anal. At. Spectrom., 2015, 30, 1469-1489, DOI: 10.1039/c4ja00491d.

11 Y. Ranebo, P. M. L. Hedberg, M. J. Whitehouse, K. Ingeneri and S. Littmann, Improved isotopic SIMS measurements of uranium particles for nuclear safeguard purposes, J. Anal. At. Spectrom., 2009, 24, 277-287.

12 P. M. L. Hedberg, P. Peres, J. B. Cliff, F. Rabemananjara, S. Littmann, H. Thiele, C. Vincent and N. Albert, Improved Particle Location and Isotopic Screening Measurements of Sub Micron Sized Particles by Secondary Ion Mass Spectrometry, J. Anal. At. Spectrom., 2011, 26, 406-413.

13 P. Peres, P. M. L. Hedberg, F. Rabemananjara, J. B. Cliff, S. Littmann, N. Albert and C. Vincent, Nuclear Safeguards and Nuclear Forensic Analysis by Secondary Ion Mass Spectrometry, 2011, ESARDA proceedings, Budapest, Hungary.

14 S. Richter and S. A. Goldberg, Improved techniques for high accuracy isotope ratio measurements of nuclear materials using thermal ionization mass spectrometry, Int. J. Mass Spectrom., 2003, 229, 181-197.

15 F. Esaka, K. Watanabe, H. Fukuyama, T. Onodera, K. T. Esaka, M. Magara, S. Sakurai and S. Usuda, Efficient Isotope Ratio Analysis of Uranium Particles in Swipe Samples by Total-Reflection X-ray Fluorescence Spectrometry and Secondary Ion Mass Spectrometry, J. Nucl. Sci. Technol., 2004, 41(11), 1027-1032.

16 K. J. Coakley, D. S. Simons and A. M. Leifer, Secondary ion mass spectrometry measurements of isotopic ratios: correction for time varying count rate, Int. J. Mass Spectrom., 2005, 240, 107-120.

17 A. D. Cutter, K. L. Hunter, P. J. K. Paterson and R. W. Stresau, The "aging" mechanism in electron multipliers and operating life, 42nd ASMS Conference on Mass Spectroscopy and Allied Topics, Chicago, Illinois, May 29-June 3, 1994.

18 G. Slodzian, M. Chaintreau, R. Dennebouy and A. Rousse, Precise in situ measurements of isotopic abundances with pulse counting of sputtered ions, Eur. Phys. J.: Appl. Phys., 2001, 14, 199-231.

19 D. L. Hoffmann, D. A. Richards, T. R. Elliott, P. L. Smart, C. D. Coath and C. J. Hawkesworth, Characterisation of secondary electron multiplier nonlinearity using MCICPMS, Int. J. Mass Spectrom., 2005, 244, 97-108.

20 J. Truyens, E. Stefaniak, S. Mialle and Y. Aregbe, NUSIMEP-7: Uranium Isotope Amount Ratios in Uranium Particles Interlaboratory Comparison Report, EUR 25179 EN, European Union 2011, DOI: 10.2787/57332. 